\title{
Correction to: Effect of longevity genetic variants on the molecular aging rate
}

\author{
Anastasia Gurinovich • Zeyuan Song • William Zhang • Anthony Federico • \\ Stefano Monti · Stacy L. Andersen • Lori L. Jennings · David J. Glass • \\ Nir Barzilai · Sofiya Millman · Thomas T. Perls • Paola Sebastiani (C)
}

Published online: 18 June 2021

(C) American Aging Association 2021

The original version of this article unfortunately contained an error in ESM2.

The corrected ESM 2 is shown online.

Publisher's note Springer Nature remains neutral with regard to jurisdictional claims in published maps and institutional affiliations.

Supplementary Information The online version contains supplementary material available at https://doi. org/10.1007/s11357-021-00400-7.

The original article can be found online at https://doi.org/ 10.1007/s11357-021-00376-4.

A. Gurinovich $\cdot$ Z. Song

Department of Biostatistics, Boston University School of Public Health, Boston, MA, USA

W. Zhang $\cdot$ N. Barzilai $\cdot$ S. Millman Institute for Aging Research, Albert Einstein College of Medicine, Bronx, NY, USA

\section{A. Federico $\cdot$ S. Monti}

Bioinformatics Program, Boston University, Boston, MA, USA

\section{S. Monti}

Division of Computational Biomedicine, Department of Medicine, Boston University School of Medicine, Boston, MA, USA

S. L. Andersen · T. T. Perls

Department of Medicine, Geriatrics Section, Boston

University School of Medicine, Boston, MA, USA

\author{
L. L. Jennings \\ Novartis Institutes for Biomedical Research, 22 Windsor \\ Street, Cambridge, MA 02139, USA \\ e-mail: lori.jennings@novartis.com \\ D. J. Glass \\ Regeneron Pharmaceuticals, 777 Old Saw Mill River Rd, \\ Tarrytown, NY 10591, USA \\ e-mail: david.glass@ regeneron.com \\ P. Sebastiani $(\bowtie)$ \\ Institute for Clinical Research and Health Policy Studies, \\ Tufts Medical Center, Boston, MA, USA \\ e-mail: psebastiani@tuftsmedicalcenter.org
}

